\title{
Cervical Carcinoma
}

National Cancer Institute

\section{Source}

National Cancer Institute. Cervical Carcinoma. NCI Thesaurus. Code C9039.

A carcinoma arising from either the exocervical squamous epithelium or the endocervical glandular epithelium. The major histologic types of cervical carcinoma are: squamous carcinoma, adenocarcinoma, adenosquamous carcinoma, adenoid cystic carcinoma and undifferentiated carcinoma. 